\title{
Live Birth Rate Comparison Between Single vs. Double Ovary Women With Assisted Reproduction: A Single Tertiary Center Study
}

Ghadeer L. Aljahdali 1, 2 , Fatimah A. Alkhaldi ${ }^{3,2}$, , Sarah F. Almujarri ${ }^{3,}, 2$, Haifa F. Alsadhan ${ }^{3,2}$, Amirah S. Yaqoub $^{3,2}$, Jawaher A. Alsahabi ${ }^{4,2}$, Nazish Masud ${ }^{5,2}$, Afaf A. Felemban 6, 2

1. College of Medicine, King Saud bin Abdulaziz University for Health Sciences, Riyadh, SAU 2. Research, King Abdullah International Medical Research Center, Riyadh, SAU 3. College of Medicine, King Saud Bin Abdulaziz University for Health Sciences, Riyadh, SAU 4. Department of Obstetrics and Gynecology, King Abdulaziz Medical City, Riyadh, SAU 5. Research Unit, King Saud Bin Abdulaziz University for Health Sciences, Riyadh, SAU 6. Department of In-Vitro Fertilization, King Abdulaziz Medical City, Riyadh, SAU

Corresponding author: Ghadeer L. Aljahdali, ghadeeraljahdali@gmail.com

\section{Abstract}

\section{Introduction}

One of the major hardships faced by married couples is the inability to conceive a child. This issue is becoming more prevalent given the increasing rate of infertility worldwide. Assisted reproductive technology (ART) has brought hope to infertile couples. We aim to estimate the live birth rate (LBR) and pregnancy rate in women with one ovary compared with those with two ovaries.

\section{Methods}

A retrospective cohort study of women who underwent ART at King Abdulaziz Medical City (Jan 2000 - Dec 2018) was conducted. Five cycles of patient data were collected. The LBR (both conditional and cumulative) was compared between women with one and two ovaries.

\section{Results}

The final analysis included 403 women. Of these, $9 \%(n=37)$ had one ovary. The majority $(59 \%, n=233)$ had primary infertility. A male-associated factor accounted for $52 \%(n=208)$ of the infertility cases. The total number of live births was 164 ; and the overall LBR from five cycles was estimated as $9 \%, 16 \%, 18 \%, 18 \%$, and $15 \%$, respectively. In the double ovary group, the highest rate was in the fourth cycle [19\% (12-26)], while in the single ovary group peaked in the third cycle [27\% (9-46)]. Pregnancy was at its highest in the first cycle, accounting for 88 pregnancies.

\section{Conclusion}

Review began 04/01/2021 Review ended 04/22/2021 Published 05/06/2021

\section{๑) Copyright 2021}

Aljahdali et al. This is an open access article distributed under the terms of the Creative Commons Attribution License CC-BY 4.0., which permits unrestricted use, distribution, and reproduction in any medium, provided the original author and source are credited.

The outcomes of ART varied between study groups. LBR was lower in single ovary women. The average of five cycles in the single and double ovary groups was $13 \%$ and $15 \%$, respectively. Nevertheless, there was no significant difference in LBR between single or double ovary women.

Categories: Obstetrics/Gynecology

Keywords: live birth rate, reproductive endocrinology, in vitro fertilization iv, single ovary

\section{Introduction}

Infertility is "a disease characterized by the failure to establish a clinical pregnancy after 12 months of regular, unprotected sexual intercourse or due to an impairment of a person's capacity to reproduce either as an individual or with his/her partner" [1]. Infertility is common worldwide with a prevalence of 8-12\% [2]. According to the World Health Organization, there has been a significant worldwide decline in fertility rates over the past few decades. The World Bank's database also shows a drastic decline in fertility rates from 5.05\% in 1964 to $2.43 \%$ in 2017 [3]. Regional statistics in Saudi Arabia indicate a decline in fertility rates from $7.25 \%$ in 1994 to $2.37 \%$ in 2017 [3]. Infertile couples tend to seek help via assisted reproductive technologies (ART) such as in vitro fertilization (IVF), intra-cytoplasmic injection (ICSI), ovulation induction (OI), and intrauterine insemination (IUI). A Brazilian study conducted in 2019 estimated the prevalence of IVF and ICSI was $70.6 \%$ and $11.7 \%$, respectively, compared to other technologies [4]. As infertility rates increasing globally, increased demand for ART has been observed. Providing patients with realistic expectations in terms of potential outcomes are mandatory before they go through the rigors of ART. The main goal of using ART is to conceive a live newborn and the probability of doing so is reflected upon the live birth rate (LBR).

Assisted reproductive technologies suitability and outcomes are dependent on many factors including age, 
type of infertility, sperm characteristics, hormone levels, history of gynecological pathologies and procedures, body mass index (BMI), and previous ART complications [5]. Moreover, conditions such as endometriosis, ovarian mass or cyst (benign or malignant), and complications (e.g., ectopic pregnancy) predispose to invasive procedures such as oophorectomy, salpingectomy, and hysterectomy which could affect IVF cycle outcomes and the length of treatment [6]. Locally, a study in Jeddah, identified the indications for laparoscopy as infertility (40\%), ectopic pregnancy (15.8\%), and endometriosis (11.1\%) [7]. These will also affect ART outcomes such as pregnancy rate and LBRs, which are of particular concern when treating women with a single ovary. It remains unclear whether there are any ramifications in terms of ART outcomes. Among the scarce data comparing women with one and two ovaries, some studies have reported that the pregnancy rate is similar between the two groups, while others have found it to be less in single ovary women [8-9]. Similarly, others reported lower LBRs among women who underwent a unilateral oophorectomy (18.6\%) compared with women who have two functional ovaries (25.4\%) [10].

Assisted reproductive technologies outcomes include LBR, cumulative LBR (CLBR), and pregnancy rate (PR). Each rate has its importance and implications. Success rates for ART cycles have traditionally been reported as pregnancies per cycle according to maternal age. In most cases, success reporting in ART involves calculating outcome parameters such as pregnancy, delivery, (live) birth per started cycle, per (oocyte) aspiration, or per (embryo) transfer. LBR is an outcome that manifests the success of IVF and ICSI; furthermore, it is the primary outcome of the number of deliveries that result in live-born neonates [11]. The CDC reported that 284,385 ART cycles were performed in 2017, resulting in 68,908 live births in the United States [12].

In Saudi Arabia, there is a huge effort and demand for ART, yet there is a vast knowledge gap that must be bridged. We need a comprehensive study assessing ART success rates, techniques, and risk factors. Our study aimed to compare pregnancy, LBR, and CLBRs among women with single and double ovaries.

\section{Materials And Methods}

\section{Study design, area, and settings}

In this retrospective cohort study conducted at King Abdulaziz Medical City (KAMC), Riyadh, Saudi Arabia, we scrutinized the data from patients' health records (electronic and hard copy) from the In Vitro Fertilization (IVF) Unit and Health Information Management (HIM) system. This study was approved by the Institutional Review Board of King Abdullah International Medical Center (KAIMRC), Riyadh, Saudi Arabia.

\section{Study participants, sampling technique, and sample size}

We included Saudi women admitted between January 2000 and December 2018 to the IVF unit who underwent IVF or ICSI during the study period. Inclusion criteria included primary or secondary infertility, double or single ovary women, and normal hormonal levels such as prolactin or thyroid-stimulating hormone (TSH). Patients were excluded for documentation issues such as missing data. We used a stratified random sampling technique. We divided the subjects who met the inclusion criteria into two groups, double and single ovary women. In the literature, the LBRs in single and double ovary women were $18-25 \%$ and $32.3 \%$, respectively [10]. Based on these previous studies, we calculated our sample size using a power of $80 \%$ and alpha 0.05 ; we assumed unequal values by keeping P1 > P2. Therefore, the estimated sample for double and single ovary groups were 300 and 132, respectively. However, we could not reach the estimated sample size for the single ovary group.

\section{Data collection}

Data were collected in collaboration with the In Vitro Fertilization and Health Information Management (HIM) departments, both of which are responsible for maintaining up-to-date records of all assisted fertility patients. Patient data from 2000 to 2015 were obtained from paper files at HIM department, while data from 2016 to 2018 were extracted from the hospital's electronic database "BESTCare system". Patients were followed during their ART trials, and the main variables included were wife and husband demographic profiles, duration of infertility, and semen analysis for men (volume, concentration, motility, morphology, viscosity, gelatinous particles, and spermatocyte). The grouping variables were single and double ovary groups and the presence of a male-associated factor as the cause of infertility. Aside from the underlying cause of infertility, pre-cycle details included type of ART, reproductive hormone levels, clinical investigations (hysteroscopy, ultrasound, laparoscopy, hysterosalpingogram), and treatments (Clomid, follicule-stimulating hormone [FSH], gonadotropin releasing hormone $(\mathrm{GnRH})$, and human chorionic gonadotropin [hCG] supplements). Data for the first five cycles for all eligible women were extracted and analyzed.

\section{Operational definitions}

The pregnancy rate was defined as the number of pregnancies achieved ending with a viable birth with the exclusion of abortions. Live births reflect the number of deliveries that resulted in a live newborn. Three different live birth rates were analyzed: conditional, conservative, and optimal. Calculation details are provided in the supplementary file. The conditional live birth rate was calculated by dividing the number of 
live births per cycle by the number of women in that cycle. Conservative live birth rate (CLBR) is the cumulative live births over women's number in the first cycle [13-14].

\section{Statistical analysis}

After data entry was complete, the initial frequencies were run to check for any data entry deficiencies. Necessary corrections were made after accessing the original files when needed. Data were analyzed using the Statistical Package for Social Sciences (SPSS) version 22 and Microsoft Excel. Patient profiles are summarized in tables using percentages and frequencies for the categorical variables (e.g., ART, treatment, investigation, and presence of comorbidities). Numerical variables (e.g., age, number of

cycle/oocytes/embryos, infertility duration) were presented as mean and standard deviation. The two groups were based on the number of ovaries. The primary outcomes (e.g., LBR, OLBR, CLBR) were presented in tables along with standard error. The LBR was calculated by dividing the total number of women who became pregnant by the eligible women who underwent ART in the corresponding cycle. The optimal live birth rate (OLBR) included women from the first cycle and other cycles. Kaplan-Meier curves were used to report the optimal estimate of the cumulative live birth rate, whereas the Greenwood formula was used to estimate the standard error. All of the estimates are presented in figures for all patients and stratification according to their respective groups [14-16].

\section{Results}

Among women admitted to the IVF department, 846 were randomly chosen. Double and single ovary women accounted for $50 \%(n=424)$ and $49.9 \%(n=422)$, respectively. From which, 403 were included in this study, $9 \%(n=37)$ single ovary while $91 \%(n=366)$ double ovaries. Primary infertility accounted for $59 \%(n=233)$. Infertility duration ranged from 24 to 168 months. Infertility due to male-associated factors accounted for $52 \%(n=208)$. Hypothyroidism was the most prevalent comorbidity, 17\% (69). A group of women $(n=91)$ had additional anovulatory cycles. On the other hand, the husband's mean age $35 \pm 8$ years. The presence of infertility problems accounted for $34 \%(n=136)$. Comorbidities included spermatic disease $11 \%(n=44)$, testicular disease $10 \%(n=39)$. Semen analysis was also recorded in comparison between positive and negative male associated factors. Among husbands presenting with a male-associated factor, $26 \%(n=54)$ received treatment, $27 \%(n=57)$ testicular sperm aspiration (TESA). Tables $1-2$ show wife and husband demographics respectively. 


\section{Cureus}

Wife Demographics

Variables

N (\%)

Infertility type

Primary

$233(59 \%)$

Secondary

$164(41 \%)$

Infertility duration (months) Median (Q1-Q3)

48 (24-168)

Presence of male-associated factor

Absent

195 (48\%)

Present

$208(52 \%)$

Presence of female-associated factor

Motility (inhospitable)

$87(44 \%)$

Oocyte

$1(1 \%)$

Endometriosis

$15(8 \%)$

Tubular

$53(27 \%)$

Uterine

$11(6 \%)$

Hormonal

$5(3 \%)$

Unexplained

$25(13 \%)$

Ovarian

Ovary Number

Single

$37(9 \%)$

Double

$366(91 \%)$

Surgery Type*

Salpingoophrectomy

Salpingectomy

Oopherectomy

$5(39 \%)$

Partial oophorectomy

$1(8 \%)$

Wife Comorbidities ${ }^{* *}$

Hypothyroidism

$69(17 \%)$

Hyperprolactinemia

$31(8 \%)$

Dyslipidaemia

$5(1 \%)$

Diabetes

$16(4 \%)$

Hypertension

$5(1 \%)$

Bronchial asthma

$10(3 \%)$

Others

$9(4 \%)$

\section{TABLE 1: Baseline Characteristics - Wife}

*From single ovary group.

*^Values reported based on the categorical variable "Yes". 


\section{Cureus}

\begin{tabular}{|c|c|c|}
\hline \multicolumn{3}{|l|}{ Husband Demographics } \\
\hline Variables & All Samples [N (\%) / Mean \pm SD] & Male-associated factor positive ${ }^{* \star}[\mathrm{N}(\%) /$ Mean $\pm \mathrm{SD}]$ \\
\hline Age (years) & $35 \pm 8$ & $36 \pm 9$ \\
\hline Comorbidities $^{*}$ & $44(11 \%)$ & $40(19 \%)$ \\
\hline Spermatic disease & $39(10 \%)$ & $30(14 \%)$ \\
\hline Testicular disease & $19(5 \%)$ & $13(6 \%)$ \\
\hline Diabetes & $14(4 \%)$ & $12(6 \%)$ \\
\hline Hypertension & $12(3 \%)$ & $10(5 \%)$ \\
\hline Others & $44(11 \%)$ & $40(19 \%)$ \\
\hline Infertility problem* & $136(34 \%)$ & $128(62 \%)$ \\
\hline Sperm motility ( $n=275)$ & $53 \pm 32$ & $36 \pm 28$ \\
\hline Normal morphology $(n=160)$ & $6 \pm 10$ & $4 \pm 9$ \\
\hline Viscosity ( $n=52)$ & $0.3 \pm 1$ & $0.3 \pm 1$ \\
\hline Concentration $(n=313)$ & $62 \pm 108$ & $22 \pm 43$ \\
\hline Volume $(n=310)$ & $3 \pm 3$ & $2 \pm 1$ \\
\hline Abstinence days $(n=125)$ & $4 \pm 2$ & $4 \pm 1$ \\
\hline Spermatocytes $(n=53)$ & $2 \pm 3$ & $2 \pm 4$ \\
\hline Leukocytes $(n=51)$ & $1 \pm 2$ & $1 \pm 1$ \\
\hline Received treatment ${ }^{*}$ & $55(14 \%)$ & $54(26 \%)$ \\
\hline Testicular sperm aspiration ${ }^{*}$ & $59(15 \%)$ & $57(27 \%)$ \\
\hline Testicular sperm extraction ${ }^{*}$ & $7(2 \%)$ & $7(3 \%)$ \\
\hline
\end{tabular}

\section{TABLE 2: Baseline characteristics - husband}

"Values reported based on categorical variable "Yes".

${ }^{* *}$ Values reported are based for male-associated factor positive patients only. Each variable considers a different denominator to calculate the percentages.

For the first cycle, the mean age and BMI were $30 \pm 5$ years and $29 \pm 5$, respectively. FSH supplementation was given to 320 women which can be sub-divided into: $72 \%(n=231)$ human menopausal gonadotropin (HMG), 20\% ( $n=65)$ Gonal F, 7\% ( $n=23)$ Puragon. In addition, gonadotropin supplementation was given to 239 of the patients: $57 \%(\mathrm{n}=135)$ Decapeptil; 30\% $(\mathrm{n}=71)$ Cetrotide; $13 \%(\mathrm{n}=31)$ Lupron. The type of ART used was $54 \%(n=208)$ ICSI, 37\% $(n=45)$ IVF, and $9 \%(n=35)$ split. The mean number of oocytes was $(10 \pm$ 6), cryopreserved ( $7 \pm 2$ ), and embryo transfer $(3 \pm 1)$. Please refer to Table 3 for further information regarding cycles one to five for all women. 


\begin{tabular}{|c|c|c|c|c|c|c|c|c|c|c|}
\hline \multirow{2}{*}{ Variable } & \multicolumn{2}{|c|}{ Cycle $1(n=397)$} & \multicolumn{2}{|c|}{ Cycle $2(n=310)$} & \multicolumn{2}{|c|}{ Cycle $3(n=219)$} & \multicolumn{2}{|c|}{ Cycle $4(n=140)$} & \multicolumn{2}{|c|}{ Cycle $5(n=88)$} \\
\hline & $\mathrm{N}$ & $\begin{array}{l}\text { Mean } \pm \\
\text { SD/\% }\end{array}$ & $\mathrm{N}$ & $\begin{array}{l}\text { Mean } \pm \\
\text { SD/\% }\end{array}$ & $\mathrm{N}$ & $\begin{array}{l}\text { Mean } \pm \\
\text { SD/\% }\end{array}$ & $\mathrm{N}$ & $\begin{array}{l}\text { Mean } \pm \\
\text { SD/\% }\end{array}$ & $\mathrm{N}$ & $\begin{array}{l}\text { Mean } \pm \\
\text { SD/\% }\end{array}$ \\
\hline Age & 376 & $30 \pm 5$ & 127 & $31 \pm 5$ & 203 & $32 \pm 5$ & 132 & $33 \pm 5$ & 79 & $34 \pm 4$ \\
\hline BMI & 339 & $29 \pm 5$ & 149 & $29 \pm 5$ & 170 & $29 \pm 5$ & 55 & $31 \pm 5$ & 35 & $31 \pm 6$ \\
\hline Number of Oocytes & 371 & $10 \pm 6$ & 286 & $10 \pm 6$ & 195 & $11 \pm 7$ & 123 & $10 \pm 6$ & 81 & $9 \pm 6$ \\
\hline $\begin{array}{l}\text { Number of Embryos } \\
\text { Transferred }\end{array}$ & 350 & $3 \pm 1$ & 269 & $3 \pm 1$ & 185 & $3 \pm 1$ & 114 & $3 \pm 1$ & 76 & $2 \pm 1$ \\
\hline Type of FSH & \multicolumn{2}{|l|}{ (320) } & \multicolumn{2}{|l|}{ (260) } & \multicolumn{2}{|c|}{ (171) } & \multicolumn{2}{|c|}{ (105) } & \multicolumn{2}{|l|}{ (73) } \\
\hline Gonal F & 65 & $20 \%$ & 51 & $20 \%$ & 36 & $21 \%$ & 13 & $12 \%$ & 13 & $18 \%$ \\
\hline HMG & 231 & $72 \%$ & 184 & $71 \%$ & 47 & $68 \%$ & 82 & $78 \%$ & 52 & $71 \%$ \\
\hline Puragon & 23 & $7 \%$ & 24 & $9 \%$ & 18 & $11 \%$ & 9 & $9 \%$ & 5 & $7 \%$ \\
\hline Bravelle & 1 & $0 \%$ & 1 & $0 \%$ & 1 & $1 \%$ & 1 & $1 \%$ & 3 & $4 \%$ \\
\hline Type of GnRH & \multicolumn{2}{|l|}{ (239) } & \multicolumn{2}{|l|}{ (203) } & \multicolumn{2}{|c|}{ (135) } & \multicolumn{2}{|l|}{ (87) } & \multicolumn{2}{|l|}{ (58) } \\
\hline Lupron & 31 & $13 \%$ & 34 & $16 \%$ & 13 & $10 \%$ & 11 & $13 \%$ & 6 & $10 \%$ \\
\hline Decapeptil & 135 & $57 \%$ & 114 & $56 \%$ & 81 & $60 \%$ & 49 & $56 \%$ & 29 & $50 \%$ \\
\hline Cetrotide & 71 & $30 \%$ & 52 & $26 \%$ & 38 & $28 \%$ & 26 & $30 \%$ & 23 & $40 \%$ \\
\hline Cycle Status $^{\star}$ & \multicolumn{2}{|l|}{ (397) } & \multicolumn{2}{|l|}{ (309) } & \multicolumn{2}{|c|}{ (218) } & \multicolumn{2}{|c|}{ (197) } & \multicolumn{2}{|l|}{ (87) } \\
\hline Done & 358 & $90 \%$ & 282 & $91 \%$ & 191 & $88 \%$ & 181 & $92 \%$ & 82 & $94 \%$ \\
\hline Cancelled & 11 & $3 \%$ & 6 & $2 \%$ & 6 & $3 \%$ & 7 & $4 \%$ & -- & -- \\
\hline Failed & 28 & $7 \%$ & 21 & $7 \%$ & 21 & $10 \%$ & 9 & $5 \%$ & 5 & $6 \%$ \\
\hline
\end{tabular}

\section{TABLE 3: Clinical characteristics of all women based on each IVF cycle}

*Values based on categorical variable "Yes".

Live and cumulative birth rates (LBR and CLBR) were estimated for each cycle in Table 4 . The total number of live births was 164 . The live birth rates per cycle were $9 \%, 16 \%, 18 \%, 18 \%$, and $15 \%$, respectively. In the double ovary group, the highest rate was in the fourth cycle [19\% (12-26)], while the single ovary group peaked in the third cycle [27\% (9-46)]. We found no statistical difference between LBR and the number of ovaries $(\mathrm{P}>0.05)$. Figure 1 shows a comparison between the number of pregnancies and live births in each cycle. Pregnancy was highest in the first cycle, accounting for 88 pregnancies, and lowest in the fifth cycle. The number of live births was highest in the second cycle and lowest in the fifth. In Figure 2, live, conservative, and optimal birth rates are presented in each cycle for all, double, and single ovary women. Live birth rates in both groups of women were similar in the first and second cycles and reached the highest rate for the single ovary group in the third cycle, while double ovary and all-women groups remained synchronous until the fifth cycle. In the fourth cycle, the single ovary group reached its lowest rate, which then increased in the fifth cycle. 


\section{Cureus}

\begin{tabular}{|c|c|c|c|c|c|}
\hline \multirow{2}{*}{$\begin{array}{l}\text { Cycle } \\
\text { (i) }\end{array}$} & \multirow{2}{*}{$\begin{array}{l}\text { Number of } \\
\text { women }\left(\mathrm{N}_{\mathrm{i}}\right)\end{array}$} & \multirow{2}{*}{$\begin{array}{l}\text { Live births } \\
\left(\text { LB }_{\mathrm{i}}\right)\end{array}$} & \multirow{2}{*}{$\begin{array}{l}\text { Live birth rate within each cycle, } \\
\%(95 \% \mathrm{Cl})^{\star}\end{array}$} & \multicolumn{2}{|c|}{ Cumulative birth rates across all cycles $\%(95 \% \mathrm{Cl})$} \\
\hline & & & & $\begin{array}{l}\text { Conservative estimate \% } \\
(95 \% \mathrm{Cl})^{\star *}\end{array}$ & $\begin{array}{l}\text { Optimal estimate \% } \\
(95 \% \mathrm{Cl})^{\star \star \star}\end{array}$ \\
\hline 1 & 397 & 35 & $9 \%(6-12)$ & $9 \%(6-12)$ & $9 \%(6-12)$ \\
\hline 2 & 310 & 51 & $16 \%(12-21)$ & $22 \%(18-26)$ & $24 \%(20-28)$ \\
\hline 3 & 219 & 40 & $18 \%(13-23)$ & $32 \%(27-36)$ & $38 \%(33-43)$ \\
\hline 4 & 140 & 25 & $18 \%(11-24)$ & $38 \%(33-43)$ & $49 \%(43-55)$ \\
\hline 5 & 88 & 13 & $15 \%(7-22)$ & $41 \%(37-46)$ & $56 \%(50-63)$ \\
\hline \multicolumn{6}{|c|}{ Women with single ovary } \\
\hline 1 & 36 & 4 & $11 \%(1-21)$ & $11 \%(1-21)$ & $11 \%(1-21)$ \\
\hline 2 & 28 & 4 & $14 \%(1-27)$ & $22 \%(9-36)$ & $24 \%(9-38)$ \\
\hline 3 & 22 & 6 & $27 \%(9-46)$ & $39 \%(23-55)$ & $45 \%(23-62)$ \\
\hline 4 & 9 & 0 & ----- & $39 \%(23-55)$ & $45 \%(22-67)$ \\
\hline 5 & 8 & 1 & $13 \%(-10-35)$ & $42 \%(26-58)$ & $52 \%(28-75)$ \\
\hline \multicolumn{6}{|c|}{ Women with double ovary } \\
\hline 1 & 361 & 31 & $9 \%(6-12)$ & $9 \%(6-12)$ & $9 \%(6-12)$ \\
\hline 2 & 282 & 47 & $17 \%(12-21)$ & $22 \%(17-26)$ & 24\% (19-28) \\
\hline 3 & 197 & 34 & $17 \%(12-23)$ & $31 \%(26-36)$ & $37 \%(32-42)$ \\
\hline 4 & 131 & 25 & $19 \%(12-26)$ & $38 \%(33-43)$ & $49 \%(43-55)$ \\
\hline 5 & 80 & 12 & $15 \%(7-23)$ & $41 \%(36-46)$ & $57 \%(50-63)$ \\
\hline
\end{tabular}

\section{TABLE 4: Live and cumulative birth rates across all cycles for 397 women undergoing IVF}

* Values are representing live birth rate in each cycle

${ }^{\star \star}$ Conservative estimate of the cumulative live birth rate

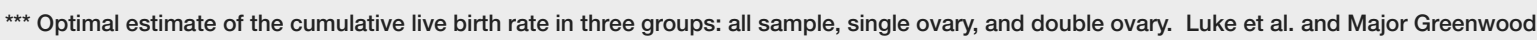
formulas were used to estimate the rates, see appendix [14-15]. 


\section{Cureus}

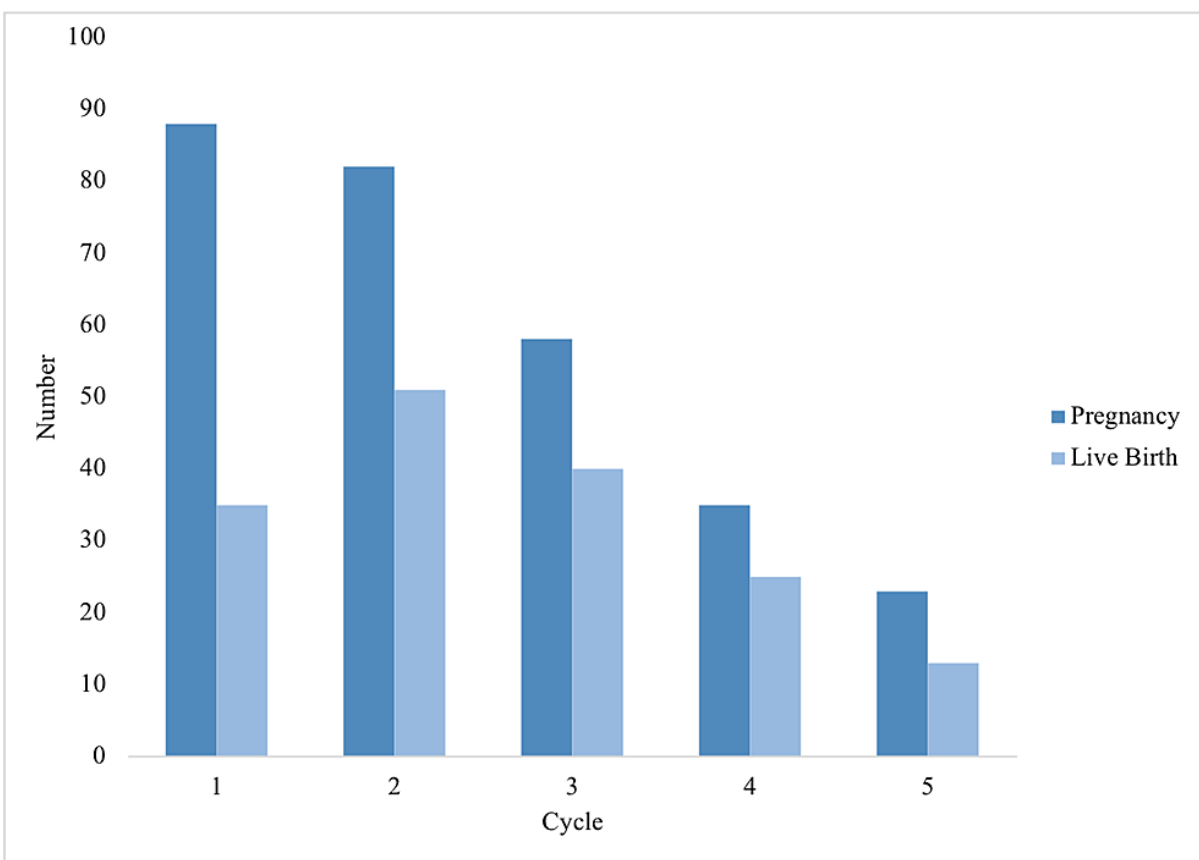

FIGURE 1: Comparison of total number of pregnancies vs. live birth across all cycles 


\section{Cureus}

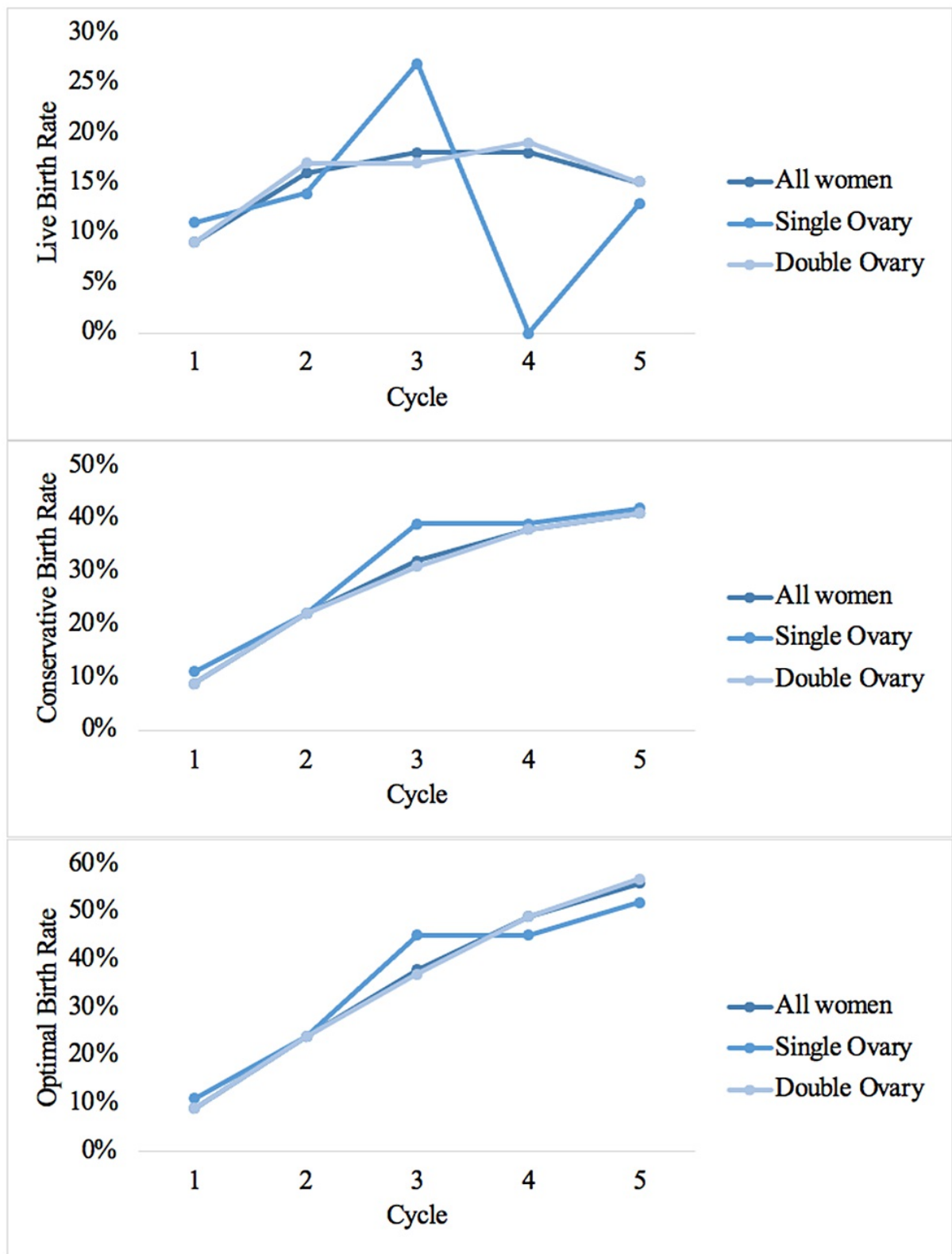

FIGURE 2: Live birth, conservative, and optimal birth rates in women with single vs. double ovary and all women

\section{Discussion}

Assisted reproductive technology (ART) has become the predominant treatment for infertility, leading to an increase in the number of infants born in most developed countries over the past 30 years. Many measures are used to express the effectiveness of ART, including LBR, defined as the live birth of a baby over $500 \mathrm{~g}$ or over 22 weeks of gestation if birth weight is unknown (deliveries of multiple pregnancies count as one live birth) [1]. Our study concentrates on the LBR after each cycle of treatment.

Both pregnancy rates and live birth rates are used to predict the success of ART, yet they differ based on many factors and do not directly correlate. Several studies explore these rates separately and identify possible factors such as age, embryo transfer duration, and number of oocytes [17]. In 2017, the Centers for Disease Control and Prevention (CDC), reported an LBR that ranged from $4.2 \%$ to $54.4 \%$ based on oocyte retrieval and an LBR that ranged from $11 \%$ to $48.5 \%$ based on embryo transfer [12]. Lind et al. found reduced LBR in women with a single ovary accounted for $18.6 \%$ compared with $25.4 \%$ in the double ovary group in the first cycle [10]. In our study, the LBR was higher in the single ovary group (11\%) for the first cycle compared with the double ovary group (9\%). A local study estimated the overall pregnancy rate as $35.1 \%$ (949 pregnancies) [18]. Here we found 286 pregnancies over 18 years and the highest number reached was 88 pregnancies in the first cycle. 
In our comparison of women with one and two ovaries, the highest pregnancy rate occurred in different cycles in the two groups. However, the cycle-specific rates showed similar live birth rates among both single and double ovary women. Concordantly, a previous study also supports the argument that single ovary women have the same chances to conceive as a woman with two ovaries [19]. Similarly proven, another study underlined that the dose of HMG required, number of follicles seen upon ultrasound examination, number of obtained oocytes, number of embryos transferred, and pregnancy rates were not statistically different between both groups of women. Nevertheless, double ovary women tend to have more follicles aspirated during laparoscopy [20].

Our results demonstrated that the average age of women seeking ART was $32 \pm 5$ years, which is in accordance with findings reported by studies performed locally and internationally $[18,21]$. They were divided into primary infertility (59\%) and secondary infertility (41\%), which are also consistent with two other studies that show a higher prevalence of primary infertility [18,22]. The highest incidence of female comorbidities was hypothyroidism (17\%), followed by hyperprolactinemia (8\%). According to the literature, an early miscarriage can be caused by a decrease in thyroxin levels, and a retrospective cohort study showed non-significant differences in the CLBR between women treated for hyperprolactinemia and control groups $[21,23]$. These two findings highlight the importance of pre-cycle treatment. On the other hand, the most common male comorbidities were spermatic and testicular-related disease (19\% and 14\%). A single-center control study compared the prevalence of comorbidities between infertile and fertile men, revealing a significantly higher percentage in the infertile men, particularly hypertension (17.8\% vs. 7.1\%) [24]. A similar pattern was observed in this study (6\% vs. $4 \%$ ).

Female-associated factors accounted for $48 \%$ of the infertility causes; the most common cause was motility (44\%), followed by tubular factors (27\%), which was previously reported to be the second most common factor in female infertility [25]. Male-associated factor infertility was the most common indication for ART, accounting for $52 \%$ of the causes. This is consistent with what has been found in previous studies $[18,26]$. Semen quality is a measure of male infertility; therefore, up to $90 \%$ of infertility cases are due to sperm abnormalities, which could stem from testicular factors [27-28]. Cases with male-associated infertility factors were grouped into two categories: spermatic diseases (19\%) and testicular diseases (14\%). Women with male-associated infertility factors had the highest pregnancy rate using in vitro fertilization [29].

\section{Limitations}

This is a single center study and the generalizability of the results are restricted. However, the research provides insight into the less studied comparison of birth rates among single and double ovary women. King Abdulaziz Medical City is one of the busiest IVF centers in Saudi Arabia and receives patients from all over the country, and many patients have been lost in the final analysis due to loss of follow-up. The results of this study hold importance in the context of Saudi population. All of the estimates in this study were reported based on the number of ovaries. The different causes for infertility were not considered in the calculation of estimates as it was outside of the scope of the present study. Also, all of the patients were undergoing ART, therefore, the probability of getting pregnant irrespective of the cause of infertility was assumed to be the same for all patients. Future studies that include multiple centers in more than one region of Saudi Arabia are recommended, which will be a better reflection on current practice.

\section{Conclusions}

In conclusion, the outcomes of ART varied between studied groups and different factors contributed to the outcomes. Live birth rates were lower in single ovary women. The average across five cycles in the single and double ovary groups were estimated as $13 \%$ and $15 \%$, respectively, with higher numbers of live births recorded among those with two ovaries. There was no statistical difference in live birth rate between single and double ovary women. The difference between pregnancy and live birth numbers was drastic in the first cycle. We recommend optimizing the quality of care by increasing patient education as well as thorough observation, and regular follow-ups through the cycle and pregnancy period to prevent unfortunate events. Further studies to explore the drop in live birth rate among single ovary women are highly recommended.

\section{Appendices \\ Supplementary file}

The linking of women to each cycle was done based on the information available for each patient in their medical record files. Irrespective of the number of cycles the women had, each cycle information was extracted after checking the MRN number and counterchecking it with the date of birth and husband's name to make sure the cycles were linked with the corresponding patients. In cases where there were discrepancies, all the files were physically checked as hard copies

The estimation of the birth rate was done using the following formulas:

A. Conditional Live Birth Rate (pi) = Number of live births / Number of women 
Where LBi refers to births ending in viable babies ( $>22$ weeks of gestation), Ni is number of all eligible women for the corresponding cycle, and SE is the standard error.

B. Conservative cumulative live birth rate $(\mathrm{CCLi})=$ Cumulative live births / Number of women at 1 st cycle

$$
\begin{gathered}
=\left[\sum j=1, i \mathrm{LBj}\right] / \mathrm{Ni} \\
\mathrm{SE}(\mathrm{CCLi})=[\mathrm{CCLi} *(1-\mathrm{CCLi}) / \mathrm{N} 1]^{1 / 2}
\end{gathered}
$$

Where Ni is the number of women who had live birth from the 1st cycle to cycle i, LB is the live births, and $\mathrm{SE}$ is the standard error.

C. Optimal cumulative livebirth rate $(\mathrm{OCLi})=\mathrm{OCLi}-1+\mathrm{pi} *(1-\mathrm{OCLi}-1)$

(Product limit estimate)

$\mathrm{SE}(\mathrm{OCLi})=(1-\mathrm{OCLi}) *\left[\sum \mathrm{j}=1, \mathrm{i} \mathrm{pj} /(\mathrm{Nj}-\mathrm{LBj})\right]^{1 / 2}$

(Greenwood's formula)

Where OCLi was based on the product limit estimate also known as the Kaplan-Meier estimator.

Greenwoods's formula was used to put the SE (standard error) on the Kaplan Meier estimator [14-15].

\section{Additional Information \\ Disclosures}

Human subjects: Consent was obtained or waived by all participants in this study. King Abdullah International Medical Research Center Institutional Review Board issued approval SP19/075/R. Animal subjects: All authors have confirmed that this study did not involve animal subjects or tissue. Conflicts of interest: In compliance with the ICMJE uniform disclosure form, all authors declare the following: Payment/services info: All authors have declared that no financial support was received from any organization for the submitted work. Financial relationships: All authors have declared that they have no financial relationships at present or within the previous three years with any organizations that might have an interest in the submitted work. Other relationships: All authors have declared that there are no other relationships or activities that could appear to have influenced the submitted work.

\section{Acknowledgements}

We would like to express our gratitude to the Research Unit faculty and the IVF department for their great efforts in this study. In particular, we would like to thank Dr. Emad Masuadi, Dr. Aamir Omair, and Ms. Rashpal Gill for their valuable assistance in the results and analysis.

\section{References}

1. Zegers-Hochschild F, Adamson GD, Dyer S, et al.: The international glossary on infertility and fertility care, 2017. Fertil Steril. 2017, 108:393-406. 10.1016/j.fertnstert.2017.06.005

2. Vander Borght M, Wyns C: Fertility and infertility: definition and epidemiology. Clin Biochem. 2018, 62:210. 10.1016/j.clinbiochem.2018.03.012

3. The World Bank. Fertility rate, total (births per woman) . (2018). https://data.worldbank.org/indicator/SP.DYN.TFRT.IN.

4. Ishikawa M: Assisted reproductive technology; IVF-ET [Article in Japanese]. Hokkaido Igaku Zasshi. 1995, 70:247-52.

5. Bhandari HM, Choudhary MK, Stewart JA: Complications of assisted reproductive technology treatment and the factors influencing reproductive outcome. Obstet Gynaecol. 2018, 20:177-86.

6. de Carvalho BR, Rosa e Silva AC, Rosa e Silva JC, dos Reis RM, Ferriani RA, Silva de Sá MF: Ovarian reserve evaluation: state of the art. J Assist Reprod Genet. 2008, 25:311-22. 10.1007/s10815-008-9241-2

7. Rouzi AA, Sahly N, Kafy S, Sawan D, Abduljabbar H: Prevalence of endometriosis at a university hospital in Jeddah, Saudi Arabia. Clin Exp Obstet Gynecol. 2015, 42:785-6.

8. Khan Z, Gada RP, Tabbaa ZM, Laughlin-Tommaso SK, Jensen JR, Coddington CC 3rd, Stewart EA: Unilateral oophorectomy results in compensatory follicular recruitment in the remaining ovary at time of ovarian 
stimulation for in vitro fertilization. Fertil Steril. 2014, 101:722-7. 10.1016/j.fertnstert.2013.11.019

9. Taheripanah R, Zamaniyan M, Meybodi MK, Amir-Arjmand MH, Mansouri A, Taheripanah A, Malih N: Are intra follicular estradiol and oocytes quality in women undergoing assisted reproductive technology different between the right and left ovaries? An observational study. Eur J Obstet Gynecol Reprod Biol X. 2019, 3:100047. 10.1016/j.eurox.2019.100047

10. Lind T, Holte J, Olofsson JI, et al.: Reduced live-birth rates after IVF/ICSI in women with previous unilateral oophorectomy: results of a multicentre cohort study. Hum Reprod. 2018, 33:238-47.

10.1093/humrep/dex358

11. De Neubourg D, Bogaerts K, Blockeel C, et al.: How do cumulative live birth rates and cumulative multiple live birth rates over complete courses of assisted reproductive technology treatment per woman compare among registries?. Hum Reprod. 2016, 31:93-9. 10.1093/humrep/dev270

12. Department of Health U, Services Centers for Disease Control H. 2017 Assisted reproductive technology fertility clinic success rates report. (2017). Accessed: April 27, 2021: http://www.cdc.gov/art/reports/.

13. Abuzeid MI, Bolonduro O, La Chance J, et al.: Cumulative live birth rate and assisted reproduction: impact of female age and transfer day. 2014.

14. Luke B, Brown MB, Wantman E, et al.: Cumulative birth rates with linked assisted reproductive technology cycles. N Engl J Med. 2012, 366:2483-91. 10.1056/NEJMoa1110238

15. Greenwood M Jr: The natural duration of cancer . Reports of Public Health and Related Subjects. 1926, 33:iv26.

16. Smith AD, Tilling K, Nelson SM, Lawlor DA: Live-birth rate associated with repeat in vitro fertilization treatment cycles. JAMA. 2015, 314:2654-62. 10.1001/jama.2015.17296

17. Cetin MT, Kumtepe Y, Kiran H, Seydaoglu G: Factors affecting pregnancy in IVF: age and duration of embryo transfer. Reprod Biomed Online. 2010, 20:380-6. 10.1016/j.rbmo.2009.12.008

18. Almaslami F, Aljunid SM, Ghailan K: Demographic determinants and outcome of in vitro fertilization (IVF) services in Saudi Arabia. J Int Med Res. 2018, 46:1537-44. 10.1177/0300060517749329

19. Lass A: The fertility potential of women with a single ovary . 1999. 10.1093/humupd/5.5.546

20. Alper MM, Seibel MM, Oskowitz SP, Smith BD, Ransil BJ, Taymor ML: Comparison of follicular response in patients with one or two ovaries in a program of in vitro fertilization. Fertil Steril. 1985, 44:652-5. 10.1016/s0015-0282(16)48982-X

21. Duan Y, Liu X, Hou W, et al.: No impact of treated hyperprolactinemia on cumulative live birth rate and perinatal outcomes in in vitro fertilization-embryo transfer. J Obstet Gynaecol Res. 2019, 45:1236-44. 10.1111/jog.13957

22. Al-Turki HA: Prevalence of primary and secondary infertility from tertiary center in eastern Saudi Arabia . Middle East Fertil Soc J. 2015, 20:237-40. 10.1016/j.mefs.2015.02.001

23. Kawwass JF, Crawford S, Session DR, Kissin DM, Jamieson DJ: Endometriosis and assisted reproductive technology: United States trends and outcomes 2000-2011. Fertil Steril. 2015, 103:1537-43. 10.1016/j.fertnstert.2015.03.003

24. Shiraishi K, Matsuyama H: Effects of medical comorbidity on male infertility and comorbidity treatment on spermatogenesis. Fertil Steril. 2018, 110:1006-1011.e2. 10.1016/j.fertnstert.2018.07.002

25. Kumar P, Mohan S, Talwar P, Rai S, Nagaraja N, Sharma P: Diagnostic office vaginohysteroscopy in evaluation of infertility prior to IVF: a retrospective analysis of 1000 cases. J Obstet Gynaecol India. 2017, 67:275-81. 10.1007/s13224-017-0972-9

26. Stern JE, Luke B, Tobias M, Gopal D, Hornstein MD, Diop H: Adverse pregnancy and birth outcomes associated with underlying diagnosis with and without assisted reproductive technology treatment. Fertil Steril. 2015, 103:1438-45. 10.1016/j.fertnstert.2015.02.027

27. Glazer CH, Bonde JP, Giwercman A, Vassard D, Pinborg A, Schmidt L, Vaclavik Bräuner E: Risk of diabetes according to male factor infertility: a register-based cohort study. Hum Reprod. 2017, 32:1474-81. 10.1093/humrep/dex097

28. Sabra SM, Al-Harbi MS: An influential relationship of seminal fluid microbial infections and infertility, Taif Region, KSA. World J Med Sci. 2014, 10:32-7.

29. Mukhtar HB, Shaman A, Mirghani HO, Almasalmah AA: The outcome of assisted reproductive techniques among couples with male factors at Prince Khalid Bin Sultan fertility centre, Kingdom of Saudi Arabia. Open Access Maced J Med Sci. 2017, 5:603-7. 10.3889/oamjms.2017.102 